\title{
One year of Methods Primers
}

\author{
Join us in celebrating the first anniversary of Nature Reviews Methods Primers.
}

We launched Nature Reviews Methods Primers in January 2021 with the goal of giving researchers a one-stop shop for everything they need to know when using a method for the first time. There are many methods journals out there, but we felt that the community was missing a journal that publishes broad introductory-level overviews of methods specifically for a non-expert audience that outline the key considerations for each method, from experimental design and data analysis to minimum reporting standards and getting around limitations.

Today, we celebrate the journal's first anniversary. To say that launching a journal during a pandemic is challenging would be an understatement. However, in our first year, we have had the opportunity to work with supremely talented researchers who jumped at the chance to pass on their methodological expertise to readers. Launching this journal would not have been possible without the hard work of our authors and peer reviewers, and we want to thank you for your time and energy in helping us build this community during such a challenging time!

Over the past year, we aimed to give readers a sense of what we are trying to accomplish at Nature Reviews Methods Primers. We have published Primers on Bayesian statistics and modelling, Genome-wide association studies, DNA origami, C-H activation, Network analysis of multivariate data in psychological sciences, Radiocarbon dating and Adaptive optics for high-resolution imaging, among many others. This wide range of topics illustrates that we are keen on serving researchers in the life and physical sciences, as well as in the psychological, social and geological sciences. And with more than 325,000 article views and 300 citations (and counting), it seems safe to say that we have been serving our readers better than we had hoped. We have an even wider range of topics coming up in 2022, so make sure you register for e-alerts and follow us on social media (@MethodsPrimers) so you do not miss a thing.

When we launched, we promised to publish Primers that bridge the gap between methods straddling different disciplines ${ }^{1}$. To this end, one of our most highly accessed, shared and cited Primers, X-ray computed tomography, covers the method's use in materials, life, food and paleontological sciences, and has been cited by researchers working in plant imaging ${ }^{2}$ and imaging of microstructures in face masks to protect against COVID-19 (REF. ${ }^{3}$ ), among others. Methods Primers have facilitated new collaborations among our authors and, we hope, the broader community as well.

We also took aim at the 'reproducibility crisis' in research, promising to deliver in each Methods Primer the most up-to-date information available on using a method, correctly analysing and interpreting data and ensuring data and metadata are shared in a way that promotes reuse and reproducibility. A few of our Primers have included new initiatives to enhance reproducibility and data sharing in their fields, including refined checklists for minimum reporting standards ${ }^{4}$ and the development of data-sharing platforms ${ }^{5}$. We hope that initiatives such as these will drive open science in their respective fields and beyond.

Looking ahead, our 2022 plans involve doing even more for our readers. That is why we plan to start publishing shorter articles in the coming months in the form of Comments. In these Comments, authors will explore specific technical challenges in one aspect of experimental design or data analysis for a given method and will provide guidelines for getting the best out of your research despite these challenges. Building on what we have in 2021, we hope readers will continue to trust us as a source for inspiration and information as they plan their research journey.

\footnotetext{
1. A helpful addition to the toolbox. Nat. Rev. Methods Primers 1, 9 (2021).

2. Piovesan, A. et al. X-ray computed tomography for 3D plant imaging. Trends Plant Sci. 26, 1171-1185 (2021).

3. Du, W. et al. Microstructure analysis and image-based modelling of face masks for COVID-19 virus protection. Commun. Mater. $\mathbf{2}$, 69 (2021).

4. van de Schoot, R. et al. Bayesian statistics and modelling. Nat. Rev. Methods Primers 1, 1 (2021).

5. Lee, K. S. et al. Raman microspectroscopy for microbiology. Nat. Rev.
} Methods Primers 1, 80 (2021). 